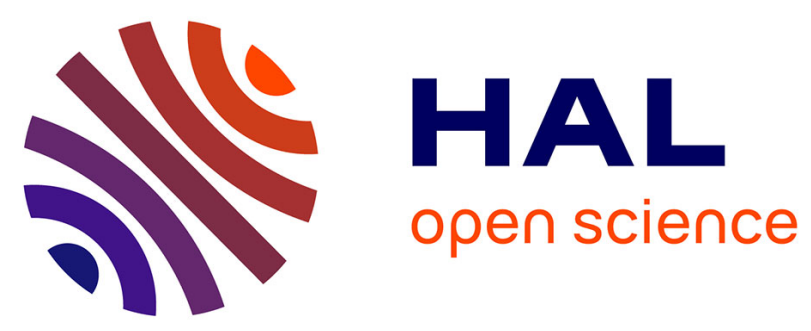

\title{
Scaling laws governing the growth and interaction of equiaxed Al-Cu dendrites: A study combining experiments with phase-field simulations
}

Ahmed Kaci Boukellal, Jean-Marc Debierre, Guillaume Reinhart, Henri Nguyen-Thi

\section{To cite this version:}

Ahmed Kaci Boukellal, Jean-Marc Debierre, Guillaume Reinhart, Henri Nguyen-Thi. Scaling laws governing the growth and interaction of equiaxed $\mathrm{Al}-\mathrm{Cu}$ dendrites: A study combining experiments with phase-field simulations. Materialia, 2018, 1, pp.62-69. 10.1016/j.mtla.2018.04.008 . hal-02083704

\author{
HAL Id: hal-02083704 \\ https://hal.science/hal-02083704
}

Submitted on 27 Oct 2021

HAL is a multi-disciplinary open access archive for the deposit and dissemination of scientific research documents, whether they are published or not. The documents may come from teaching and research institutions in France or abroad, or from public or private research centers.
L'archive ouverte pluridisciplinaire $\mathbf{H A L}$, est destinée au dépôt et à la diffusion de documents scientifiques de niveau recherche, publiés ou non, émanant des établissements d'enseignement et de recherche français ou étrangers, des laboratoires publics ou privés. 


\title{
Scaling laws governing the growth and interaction of equiaxed $\mathrm{Al}-\mathrm{Cu}$ dendrites: a study combining experiments with phase-field simulations.
}

\author{
Ahmed Kaci Boukellal, Jean-Marc Debierre, Guillaume Reinhart, Henri \\ Nguyen Thi \\ Aix-Marseille Université, CNRS, Université de Toulon, IM2NP UMR 7334, 13397 \\ Marseille, France
}

\begin{abstract}
We report on equiaxed dendritic growth in solidifying $\mathrm{Al}-\mathrm{Cu}$ alloys, particularly the interactions between the dendrites that ultimately form the grain structure in the solid. A three-dimensional phase-field code is designed to reproduce the same conditions as the ones imposed in the experiments. Our numerical results allow us to propose scaling laws that govern the dynamics of dendritic primary arms, with the elemental composition and the average distance between two nuclei as physical parameters. These laws are used to extrapolate our numerical results to the experimental situation where phasefield simulations are beyond reach. Good quantitative agreement is found between the predicted behavior and the experimental observations, which validates the scaling laws obtained.
\end{abstract}

\section{Introduction}

Aluminum-copper alloys provide the common basis of aluminum bronzes that are used in many industrial areas, such as aeronautics, automotive industry, or water supply. They are valued for their lightness, strength, hardness, and high resistance to corrosion $[1,2]$.

The fabrication of aluminum bronzes usually starts from the molten alloy that is solidified by cooling down the system. For most structural materials, homogeneous and isotropic properties are seeked. To obtain such properties, equiaxed growth under isothermal cooling conditions is usually prefered [3]. 
This solidification process occurs in two successive stages. During the nucleation stage, solid nuclei appear throughout the system, often but not always promoted by the addition of refiners [4]. In the second stage, equiaxed dendrites grow from these nuclei. At the very beginning of their growth, the solid grains can be considered isolated but, since solute is constantly rejected in the liquid, a diffusion layer progressively builds up around the grains and they soon start to interact. Equiaxed growth stops when the interactions between the arms of neighboring dendrites become strong enough [5]. This process is at the origin of the polycrystalline structure that largely conditions the physical properties of the material.

In the last decades, a number of numerical approaches were developed to describe the simultaneous growth of equiaxed dendrites from a liquid bath. Currently, the gap between small-scale and large-scale simulations in three dimensions (3D) is not yet bridged [6, 7], mainly because small-scale phase-field methods have remained limited to $2 \mathrm{D}$ so far [8]. This situation is unfavorable because in large-scale methods the kinetic of the dendrite tip is a necessary input that could be obtained from 3D small-scale simulations. The purpose of this paper is to improve the current situation by combining 3D phase-field numerical simulations with experiments to establish simple laws that govern the dynamics of two interacting equiaxed dendrites.

We consider thin samples of an initially liquid aluminum alloy containing copper at a nominal concentration $c_{0}$. Equiaxed dendrites are grown by imposing a uniform and constant cooling rate $R$. Our numerical simulations are performed in three dimensions. They are based on a phase-field model adapted to the present case of isothermal cooling. The accuracy and versatility of 3D phase-field models were recently demonstrated by quantitative comparisons with a wide variety of experimental situations [9-14].

In the present phase-field simulations, the limiting length is the capillary length that is inversely proportional to $c_{0}$. Phase-field simulations at experimental concentrations (typically $10 \mathrm{wt} \% \mathrm{Cu}$ ) are currently out of reach because of the huge number of domain points (several billions) necessary to resolve the capillary length with sufficient accuracy. This leads us to perform numerical simulations at lower concentrations and to establish scaling laws depending both on $c_{0}$ and on the average distance between two dendrites. Extrapolation to higher values of $c_{0}$ allows direct comparison with the experiments.

We remark that three-dimensional numerical simulations for high copper concentrations are in fact feasible within the dendritic needle network 
(DNN) model [15]. This approach has proved very successful to simulate large systems consisting of several columnar dendrites that grow and compete in directional solidification $[15,16]$. One requirement of the model is that dendrite tips obey the solvability condition $\rho^{2} V=$ cst, where $\rho$ is the radius of curvature and $V$ the growth velocity. However, as discussed in the following, this condition is never verified in our case, so that DNN would be less suited for the present situation of equiaxed dendrites.

Previous experimental results [5] show that collisions between adjacent dendrites occur within a minute or so, while diffusion times are much longer. The growing dendrites are thus always very far from steady-state conditions. This point was confirmed by the copper concentration measured at the solidliquid interface: values lower than $1.08 c_{0}$ were found [5], very far from the value $c_{0} / k \simeq 7.1 c_{0}$ that would be reached in a stationary state. Phasefield simulations are expected to follow the system evolution quantitatively, which makes them especially well suited to study the present solidification process despite its intrinsically transient nature. For instance, the ability of the phase-field approach to describe the initial transient for directional solidification of similar alloys was recently demonstrated [17].

Altogether, the numerical results provided by our phase-field model should give new insight into the laws governing interactions between growing equiaxed dendrite. A brief numerical study of these interactions has already been reported [18]. However, as noted by the authors, the mesoscopic model they used for this study did not allow consideration of fully transient conditions.

This paper is organized as follows. Section 2 gives a brief description of the experimental setup and presents two sets of results that provide the experimental basis of our work. Section 3 gives the main ingredients of the phase-field code used in the simulations, and in section 4 numerical results obtained for the generic features of equiaxed dendrites are discussed. Based on systematic simulations of equiaxed growth, the scaling of the tip velocity with time, copper concentration and distance between two dendrites is established and the corresponding scaling laws are presented in section 5. Direct comparisons between the experimental data and the obtained scaling laws are made in section 6 . Finally, a short summary completed by our conclusion is provided in section 7 . 


\section{Experiments}

Synchrotron X-ray radiography was used to analyze in situ the development of equiaxed grains in two samples of composition $\mathrm{Al}-4 \mathrm{wt} \% \mathrm{Cu}$ and Al-10 wt\% Cu respectively. The experiments were carried out at the ID19 beamline of ESRF (European Synchrotron Radiation Facility). The sheetlike thin samples were $40 \mathrm{~mm}$ in length, $6 \mathrm{~mm}$ in width, and about $200 \mu \mathrm{m}$ in thickness. The main surface of the sample was set perpendicular to the incident monochromatic X-ray beam with an energy $E=14 \mathrm{keV}$. Sequences of radiographs were recorded during solidification experiments with a FReLoN CCD camera providing a pixel size of $7.46 \mu \mathrm{m}$, an exposure time of $1 \mathrm{~s}$, and a field of view of $15 \mathrm{~mm} \times 6 \mathrm{~mm}$, which is sufficient to distinguish the main dendrite features during their development. Equiaxed growth is achieved in a furnace, which is described in detail elsewhere [19]. The two heating elements of the furnace are precisely adjusted to melt the samples in nearly isothermal conditions. The same cooling rate was applied on both heaters. Heterogeneous nucleation occured at the crucible wall/oxide skin, leading to the development of equiaxed grains. Most of the grains remained attached to the wall during their development as previously reported by Bogno et al. [5]. Several melting/solidification cycles with different cooling rates were applied to the samples. In this paper, we will only consider experiments carried out with a cooling rate $R=0.5 \mathrm{~K} / \mathrm{min}$.

Dendrite arms of two grains were selected to investigate the impact of solutal interactions during solidification. A radiograph recorded during their growth, along with measurements of the evolution of their growth velocity as a function of time, is shown in Figure 1.a for the Al-4 wt\% Cu sample and in Figure 1.b for the Al-10 wt\% Cu sample. These dendrite arms were chosen because their growth velocity profile clearly exhibits an initial acceleration that corresponds to a free growth regime as previously reported by Bogno et al. [5]. They also grow almost horizontally so that they are less exposed to gravity-driven fluid flow [20]. Only a limited number of dendrites develop such horizontal arms since grains nucleate with a random crystallographic orientation. The dendrite arm of the Al-4 wt\% Cu sample is facing a side of the sample and the dendrite arm of the $\mathrm{Al}-10 \mathrm{wt} \% \mathrm{Cu}$ sample is facing another dendrite arm. Both configurations lead to solutal interactions that induce a significant decrease of the growth velocity after a maximum velocity

value is reached. It is worth noting that a plateau in the growth velocity is measured at the early stage of growth for the dendrite arm of the Al-4 wt\% 
$\mathrm{Cu}$ sample. This feature does not appear in the measured velocity for the dendrite arm of the Al-10 wt\% Cu sample, although it might have occurred, but then would not have been captured due to the rather large time length between two successive recorded images $(14 \mathrm{~s})$.

\section{Phase-field model}

We make use of the thin interface phase-field model introduced by Alain Karma and co-workers [21, 22]. This model is adapted here to the experimental condition of a constant cooling rate.

Rather than the usual phase-field $\varphi \in[-1,1]$, we use the pre-conditioned phase-field

$$
\psi=\sqrt{2} \tanh ^{-1}(\varphi)
$$

as in $[10-13,23,24]$. This change of variable was introduced by Glasner [25] with the purpose of increasing the code efficiency by using coarser numerical grids without loss of accuracy. We also make use of Graphics Processing Unit (GPU) parallel programming [11-13,26] to further increase the efficiency of our code.

We only give the main lines of the model here. For more details, we refer the reader elsewhere [10]. Numerical simulations are performed in a thin parallelepipedic domain, $x$ lying along the sample width, $y$ along the sample thickness and $z$ along the sample height.

\subsection{Evolution equations}

The solid-liquid interface width $W_{0}$ is used as the unit length [27, 28]. Al- $\mathrm{Cu}$ being a rough material, a unit time $\tau_{0}=a_{0} W_{0}^{3} /\left(D d_{0}\right)$ is imposed to cancel the kinetic coefficient $[27,28]$. Here, $d_{0}$ is the chemical capillary length, $D$ the solute diffusion constant in the liquid phase (the one-sided model is considered here), and $a_{0} \simeq 0.5539$. As equiaxed growth with a constant cooling rate $R$ is considered, the imposed temperature appears in the phase field equations through a factor

$$
\Theta(t)=\left(\frac{1}{1-k}\right)\left(\frac{R t}{T_{L}-T_{M}}\right)=\frac{R t}{\beta_{k} m c_{0}},
$$

where $T_{L}$ is the alloy Liquidus temperature and $T_{M}$ the melting temperature of pure aluminium, $\beta_{k}=(1-k)$, $k$ being the partition coefficient, and $m<0$ is the Liquidus slope. The $\Theta$ factor represents the slow temperature decrease 
that is imposed in the experiments [5]. Solidification begins at the Liquidus temperature, and the sample temperature typically decreases by only a few degrees during the whole process. In order to obtain a non-dimensional version of the evolution equation for $\psi$, we follow the usual convention where lengths and times are respectively divided by $W_{0}$ and $\tau_{0}$. The resulting equation is

$$
\begin{aligned}
& \left(1-\beta_{k} \Theta\right) a_{s}^{2} \frac{\partial \psi}{\partial t}=a_{s}^{2}\left[\nabla^{2} \psi-\sqrt{2} \varphi(\vec{\nabla} \psi)^{2}\right] \\
& +\sqrt{2}\left[\varphi-\lambda\left(1-\varphi^{2}\right)(U+\Theta)\right]+2 a_{s} \vec{\nabla} a_{s} \vec{\nabla} \psi \\
& +\frac{\sqrt{2}}{\left(1-\varphi^{2}\right)} \vec{\nabla} \vec{A}
\end{aligned}
$$

As in the case of directional solidification, the temperature factor $\Theta(t)$ is also introduced on the left hand side of this equation to minimize higher-order spurious terms [22].

The nondimensional concentration field $U$ is related to the physical concentration field $c$ through

$$
c=c_{0}\left(1+\beta_{k} U\right)\left(\frac{\alpha_{k}-\beta_{k} \varphi}{2}\right)
$$

where $\alpha_{k}=(1+k)$. For our rough material, the constant that couples the non-dimensional concentration field $U$ to the phase-field is $\lambda=75 D^{*} / 47$, with $D^{*}=D \tau_{0} / W_{0}^{2}[28]$.

Both $a_{s}$ and $\vec{A}$ depend on the crystal anisotropy. In the present case, the anisotropy is cubic and the crystal axes [1 00$]$, [ [ $\left.\begin{array}{lll}0 & 1 & 0\end{array}\right]$ and [ $\left[\begin{array}{lll}0 & 0 & 1\end{array}\right]$ are aligned with the $x, y$, and $z$ axes of the numerical mesh. The corresponding anisotropy function reads then [29]

$$
a_{s}=\left(1-3 \epsilon_{4}\right)+4 \epsilon_{4}\left(n_{x}^{4}+n_{y}^{4}+n_{z}^{4}\right),
$$

where $n_{x}, n_{y}, n_{z}$ are the components of the unit vector $\vec{n}$ along the normal to the solid-liquid interface and $\epsilon_{4}$ is the anisotropy strength. The three components of the anisotropy vector $\vec{A}$ are given by

$$
A_{x, y, z}=16 \epsilon_{4} \frac{\left(1-\varphi^{2}\right)}{\sqrt{2}}|\vec{\nabla} \psi| a_{s} n_{x, y, z}\left[\left(n_{x}^{4}+n_{y}^{4}+n_{z}^{4}\right)-n_{x, y, z}^{2}\right] .
$$


As we neglect solute diffusion in the solid (one-sided model), we must introduce a corrective solute current

$$
\vec{j}_{a t}=\frac{W_{0}}{2 \sqrt{2}} c_{0} \beta_{k} G \vec{n}
$$

where

$$
G=\frac{1-\varphi^{2}}{\sqrt{2}}\left(1+\beta_{k} U\right) \frac{\partial \psi}{\partial t}
$$

in order to counterbalance the excess of solute trapping and other spurious corrections due to the finite interface thickness $W_{0}[21,22]$.

The evolution equation for the nondimensional concentration field $U$ is then $[21,22]$

$$
\begin{aligned}
\left(\alpha_{k}-\beta_{k} \varphi\right) \frac{\partial U}{\partial t}= & (1-\varphi) D^{*} \nabla^{2} U \\
& -\frac{\left(1-\varphi^{2}\right)}{\sqrt{2}} D^{*} \vec{\nabla} \psi \vec{\nabla} U \\
& -\frac{1}{\sqrt{2}} \vec{n} \vec{\nabla} G+G\left(1-\frac{\vec{\nabla} \vec{n}}{\sqrt{2}}\right),
\end{aligned}
$$

independent of the solidification type (equiaxed or directional). This scheme is equivalent to the one used in [8] to perform $2 \mathrm{D}$ simulations in similar conditions.

\subsection{Parameters and conditions imposed}

The main physical parameters used in the simulations for the aluminumcopper alloys are listed in table 1 . Let us note that the present estimate of the partition coefficient, $k=0.14$, is an average value over an extended range of concentrations $\left(c_{0}<33 \mathrm{wt} \% \mathrm{Cu}\right)[30]$. In order to obtain more precise results one should extract the small variations of $k$ with the copper concentration from a more detailed phase diagram and include them in the numerical code. We expect that the simulation results would be more accurate but the main conclusions of the present work should remain very similar. The first step consists in defining a suitable initial condition. The present choice is an equilibrium shape that is prepared by bringing an initially spherical solid nucleus (50 $\mu \mathrm{m}$ in radius) to equilibrium. The pre-conditioned phase-field $\psi$ and the dimensionless concentration field $U$ are calculated for a given temperature $T$ close to the Liquidus temperature. Then $T$ is varied up and down with a 
Table 1: Physical parameters used in the simulations.

\begin{tabular}{cccc}
\hline Parameter & physical meaning & Value & unit \\
\hline \hline$D$ & $\begin{array}{c}\text { copper diffusion coefficient } \\
\text { in the liquid }\end{array}$ & 3000.0 & $\mu \mathrm{m}^{2} / \mathrm{s}$ \\
& partition coefficient & 0.14 & - \\
\hline$k$ & Liquidus slope & -2.6 & $\mathrm{~K} / \mathrm{wt} \% \mathrm{Cu}$ \\
\hline$m$ & $\begin{array}{c}\text { aluminum melting } \\
\text { temperature }\end{array}$ & 933 & $\mathrm{~K}$ \\
\hline$T_{0}$ & copper concentration & $1.0,1.5,2.0$ & $\mathrm{wt} \% \mathrm{Cu}$ \\
\hline$c_{0}$ & crystal cubic anisotropy & 0.01 & - \\
\hline$\epsilon_{4}$ & Gibbs-Thomson coefficient & 0.236 & $\mathrm{~K} \mu \mathrm{m}$ \\
\hline$\Gamma$ & & &
\end{tabular}

progressively decreasing amplitude, until the interface velocity is arbitrarily close to zero. Once the seed in equilibrium, the growth simulation is started from there. Mirror (no-flux) conditions are constantly imposed at all the domain boundaries. These boundary conditions allow us to simulate only one half of the experimental thickness. Also, a mirror dendrite is automatically progressing toward the reference dendrite from the right and another mirror dendrite from above, causing the two primary arms of the reference dendrite to collide with their mirrors. The interface width is taken sufficiently small, $W_{0}=24.0 d_{0}$, that no spurious side-branching occurs unless a spatiotemporal noise is introduced. Figure 2 shows how the simulation results for the time evolution of the dendrite tip velocity are affected by the imposed interface thickness $W_{0}=\xi d_{0}$. A good convergence is observed when the parameter $\xi$ is decreased: as expected, the precision of the code is improved when the interface thickness is reduced. On the other hand, since the CPU/GPU time is proportional to $\xi^{-5}$, we need to increase $\xi$ to reach accessible computation times. A reasonable compromise between precision and calculation time is obtained here for the choice $\xi=24.0$.

As the numerical unit length $W_{0}$ is inversely proportional the copper concentration, the mesh grid $\delta s$ varies the same way. We take here $\delta s / W_{0}=1.0$. As shown in [25], this choice gives very accurate results for a pre-conditioned phase-field. The dimensions of the physical and numerical domains are gathered in table 2. This table implies that simulations at high concentrations are practically impossible with our code. For instance, our smallest system 
would contain $4.0 \times 10^{9}$ grid points for the concentration $c_{0}=10 \mathrm{wt} \% \mathrm{Cu}$ used in most experiments. Our strategy then consists in performing simulations at lower concentrations and in finding reliable laws to extrapolate the obtained results to higher concentrations.

Table 2: Physical dimensions of the system $\left(L_{x}=L_{z}=L\right)$ for different copper concentrations and corresponding numbers of mesh points in the numerical domain.

\begin{tabular}{ccc}
$L_{x} \times L_{y} \times L_{z}\left(\mu \mathrm{m}^{3}\right)$ & $c_{0}(\mathrm{wt} \% \mathrm{Cu})$ & $N_{x} \times N_{y} \times N_{z}$ \\
\hline \hline $800 \times 100 \times 800$ & 1 & $316 \times 40 \times 316$ \\
\hline & 1.5 & $474 \times 60 \times 474$ \\
\hline \hline $1000 \times 100 \times 1000$ & 2 & $632 \times 80 \times 632$ \\
\hline & 1 & $394 \times 40 \times 394$ \\
\hline \hline $1200 \times 100 \times 1200$ & 1.5 & $594 \times 60 \times 594$ \\
\hline & 2 & $792 \times 80 \times 792$ \\
\hline \hline & 1 & $474 \times 40 \times 474$ \\
\hline \hline
\end{tabular}

\section{Numerical results for generic features of equiaxed growth}

\subsection{Successive phases in the dendrite evolution}

In the present simulations, an eighth of the whole equilibrium solid obtained previously is taken as the initial nucleus and its center is placed at the corner of the numerical domain. Thus, only an eighth of a dendrite is simulated as shown in figure 3. Temperature is then lowered at a constant cooling rate $R=0.5 \mathrm{~K} / \mathrm{min}$, which provokes growth of two primary arms along $x$ and $z(\langle 100\rangle$ crystallographic directions). The dendrite arms grow freely at first but, due to the limited available distance $L$, they start colliding with their mirror images after some time. Three main growth phases can be distinguished (see the next subsection for a detailed description and see the movie provided as supplementary material for a visual support).

1. The initial slow growth (about 60s in figure 3) is the consequence of the initially low and slowly increasing undercooling.

2. After that, the copper diffusion fields ahead of the two impinging dendrites start to overlap, the resulting interactions progressively slow down 
the dendrites that ultimately stops when they reach the domain boundary (at about 100s in figure 3).

3. From now on, slow ripening occurs during which the tip velocity decreases sharply, the primary arms broaden, secondary arms develop and progressively occupy the whole available space, leaving highly concentrated narrow liquid channels.

Let us mention that, since we are not concerned with secondary instabilities like side-branching here, no numerical noise is imposed in the simulations discussed in the paper. We nevertheless performed a few simulations including a weak spatiotemporal noise as in [10] to test the effect of thermal fluctuations. As a result, the growth of the dendrite primary arms was basically unaffected by the noise.

\subsection{Time evolution of the dendrite physical properties}

To start with, the time evolution of a number of characteristic properties is presented and analyzed. Let us define the dendrite tip as the solid point that is most distant from the center of the initial nucleus along the horizontal axis $x$ (the $z$ tip may be alternatively chosen). We denote by $x_{t i p}, V$, and $\rho_{1}$ the tip location, tip velocity, and tip radius of curvature measured in the $x z$ plane.

Figure 4 shows the time evolution of $V$ and $\rho_{1}$. After about 30s, $V$ and $\rho_{1}$ systematically vary in opposite directions but from figure $4 \mathrm{c}$, it is clear that during the growth regime (phases 1 and $2, t<90 \mathrm{~s}$ ) the dendrite tip never reaches a stationary state where the selection parameter $\rho_{1}^{2} V$ remains constant. We found very similar results for the radius of curvature $\rho_{2}$ measured in the $x y$ plane. The fact that both radii are comparable in magnitude and in time evolution shows that confinement effects due to the crucible thickness are weak. As a consequence, the dendrite tip must be fully 3D and not 2D. This is confirmed in figure 2 that includes the results of a $2 \mathrm{D}$ simulation.

The interactions of the dendrite with the mirror dendrite that grows towards it progressively increase in time. These interactions result from an overlap between the copper diffusion fields ahead of the dendrite tips. Strong interactions can be expected at a given time $t$ if two conditions are simultaneously fulfilled:

i) the remaining distance $L-x_{\text {tip }}$ is not too large as compared to the instantaneous diffusion length $\ell_{s}=D / V$,

ii) the time remaining before the tip becomes blocked, $t_{f}-t$ is not too small as compared to the instantaneous diffusion time $t_{d}=D /\left(k V^{2}\right)$. 
The factor $1 / k$ is introduced in the expression of $t_{d}$ is appropriate for the transient starting from a uniform concentration profile $c=c_{0}$ and leading to a steady state [31].

Figure 5a shows the time evolution of the remaining distance divided by $\ell_{s}$. One sees that the curve reaches a maximum of about 2.5 at time $t \simeq 70 \mathrm{~s}$ and that the remaining time divided by $t_{d}$ almost simultaneously reaches a maximum of about 0.5 (figure $5 \mathrm{~b}$ ). In the neighborhood of these maxima, substantial tip-tip interactions are expected because it was actually shown that dendrite tips growing in opposite directions weakly (but definitely) interact when they are closer than three diffusion lengths [32]. In the present case of head-on collisions, one expects the diffusion fields to overlap much more effectively. This is confirmed by our results showing that the interaction becomes significant at the beginning of phase 2 for a tip to tip distance of about $4 \ell_{s}$.

During phase 1, the overlap between the diffusion fields remains weak, as shown by the concentration profiles in figure $5 \mathrm{c}$. This is so because the buildup of copper ahead of the tip takes much longer than the experience duration. Altogether, it takes about 60s for the diffusion fields to start overlapping. The ensuing collision stops the dendrite progression at time $t_{f} \simeq 100 \mathrm{~s}$. At later times, the dendrite tip is almost immobile and the copper concentration ahead of it continues to increases in time, as shown in figure 5c: this corresponds to the third growth phase (ripening).

\section{Scaling laws for the intermediate growth regime (phase 2)}

\subsection{Influence of two physical parameters : concentration and available dis- tance}

Figure 6 shows the dendrite tip velocity as a function of time for different copper concentrations $c_{0}$ and different values of $L$ that is half of the initial distance between two identical nuclei (or the available distance for each nucleus). Most features are common to all the chosen parameter sets $\left(L, c_{0}\right)$, like the existence of a maximum tip velocity, $V_{m}$, at a time $t_{m}$. The similarities between the different curves strongly suggest that scaling laws can be derived.

With this in mind, we focus on a velocity interval $\left[V_{m}^{\prime}, V_{m}\right]$ corresponding to a time interval $\left[t_{m}^{\prime}, t_{m}\right]$. This interval must be sufficiently far from the influence of the initial condition and still cover most of the second growth phase described in the previous subsection. For this reason, we choose $V_{m}^{\prime}=$ 
$V_{m} / 2$, so that we consider the fast growth regime that is abruptly stopped by dendrite-dendrite collision when the diffusion fields start to overlap (growth phase 2). All the $V(t)$ data obtained for the different parameter sets $\left(L, c_{0}\right)$ are gathered in figure 7 , where the scaled velocity

$$
Y=\left(2 V-V_{m}\right) / V_{m}
$$

is plotted as a function of the scaled time

$$
X=\left(t-t_{m}^{\prime}\right) / \Delta t_{m},
$$

with $\Delta t_{m}=t_{m}-t_{m}^{\prime}$. For these scaled coordinates one sees that all the data collapse on a master curve that represents the dynamical scaling law for the velocity evolution in the chosen time interval. It is to be noted that this law remains qualitative so far because the physical values of $V_{m}$ and $\Delta t_{m}$ that are of practical importance are hidden by the scaling.

\subsection{Scaling with the physical parameters}

In order to predict the values of $V_{m}$ and $\Delta t_{m}$ for a given parameter set $\left(L, c_{0}\right)$, one needs to extract additional scaling laws from the data (provided such laws exist). The growth velocity $V$ should vary as the applied undercooling $-\Theta(t)$ that is expressed in equation (2) as a function of time and concentration. On one hand, for a given concentration $c_{0}$, the maximum velocity $V_{m}$ must increase with the available distance $L$ because the undercooling is proportional to time. On the other hand, for $L$ fixed, $V_{m}$ must decrease with $c_{0}$ because the undercooling is inversely proportional to $c_{0}$. As seen in figure 6, our numerical results do comply with these requirements.

One can then expect that $V_{m}$ varies as $\left(L / c_{0}{ }^{a}\right)^{\alpha}$ with $a$ and $\alpha$ two positive exponents. As seen in figure 8, a satisfactory scaling can be obtained for simple values of the scaling exponents, $a=1 / 3$ and $\alpha=2$, thus giving the velocity law

$$
V_{m} \simeq 2.6 \times 10^{-5}\left(\frac{L^{2}}{c_{0}^{2 / 3}}\right)
$$

with $V_{m}$ in micrometers per second for $L$ in micrometers and $c_{0}$ in wt\% $\mathrm{Cu}$. These values of $a$ and $\alpha$ are obtained by numerical fits of our $V_{m}$ data, the resulting estimates being slightly rounded to give simple integer ratios.

Moreover, a scaling law for the time interval $\Delta t_{m}$ is also needed. Our numerical data indicate that, for all the concentrations considered, one has

$$
V_{m} \Delta t_{m} / L=L_{m} / L \simeq 0.546
$$


within a few percent (figure 9). The scaling law that is compatible both with this result and with equation (12) is

$$
\Delta t_{m} \simeq 2.1 \times 10^{4}\left(\frac{c_{0}^{2 / 3}}{L}\right) .
$$

It gives $\Delta t_{m}$ in seconds for $L$ in micrometers and $c_{0}$ in wt $\% \mathrm{Cu}$. Here again, a good agreement is found between this law and our numerical data for $\Delta t_{m}$ (figure 8).

\section{Comparison between numerics and experiments}

The experimental velocity curve obtained for $c_{0}=4 \mathrm{wt} \% \mathrm{Cu}$ gives $V_{m} \simeq$ $22.8 \mu \mathrm{m} / \mathrm{s}$ and $\Delta t_{m} \simeq 25.2 \mathrm{~s}$ (figure 1a). Thus $L_{m}=V_{m} \Delta t_{m} \simeq 575 \mu \mathrm{m}$, so that the available growth distance predicted by equation (13) is $L \simeq 1053 \mu \mathrm{m}$. Measuring directly the nucleus-wall distance in figure 1a, one obtains $L \simeq$ $1050 \mu \mathrm{m}$, in perfect agreement with the scaling prediction. This result is especially significant because the observed occurence of a dendrite colliding with the crucible wall is very comparable to the situation that is simulated numerically. Alternatively, for $c_{0}=10 \mathrm{wt} \% \mathrm{Cu}$, figure $1 \mathrm{~b}$ gives $V_{m} \simeq 11.1 \mu \mathrm{m} / \mathrm{s}$ and $\Delta t_{m} \simeq 68.6 \mathrm{~s}$, thus $L_{m} \simeq 760 \mu \mathrm{m}$. The available growth distance predicted by equation (13) is now $L \simeq 1390 \mu \mathrm{m}$ but measuring half the nucleus to nucleus distance in figure $1 \mathrm{~b}$ gives $L \simeq 1200 \mu \mathrm{m}$, a value about $15 \%$ lower than the scaling prediction. However, one sees that the two dendrites on the right side of figure $1 \mathrm{~b}$ are obviously at an earlier growth stage than the leftmost one. One may thus reasonably assume that the available distance $L$ for the leftmost dendrite is significantly larger than half the nucleus to nucleus distance (possibly by about 15\%). Altogether, the reasonable agreement found in both cases with the experiments gives strong support to our scaling approach.

Obviously, numerous physical effects exist in the experiments that are not included in our simple model. Two such effects are for instance :

1. In the simulations, two mirror nuclei appear simultaneously and they grow in opposite directions. In the experiments dendrites start to grow at different times, with different crystallographic orientations and different growth directions (as just discussed for figure 1b).

2. In the experiments, gravity effects provoke globally vertical solute currents that modify the copper concentration in front of the primary arms of the dendrite according to their growth direction. 
Both effects induce local perturbations in the copper concentration field that propagate on large distances and affect the shape of the dendrite tip as a consequence. One can thus expect that the prefactors and/or the exponents that enters the scaling laws (12) and (14) are modified by the two effects just mentioned. As just discussed for the experimental image in figure 1b, including these effects in the numerics is expected to improve the agreement with the experiments.

\section{Summary and conclusion}

We have obtained both experimental and numerical data for the time evolution of aluminum-copper equiaxed dendrites. Numerical estimates of the dendrite tip velocity $V$ and radius of curvature $\rho$ have shown that $\rho^{2} V$ is never constant, thus that equiaxed dendritic growth is an intrinsically transient phenomenon. Three growth phases have been identified: an initial slow growth phase, followed by a fast growth phase abruptly stopped by collisions with other dendrites, and finally a ripening phase. Our numerical data for the second growth phase have allowed us to establish the scaling laws that govern the dendrite tip maximum velocity $V_{m}$ and the time $\Delta t_{m}$ needed for the tip velocity to increase from $V_{m} / 2$ to $V_{m}$ as functions of copper concentration $c_{0}$ and available growth distance $L$. From these scaling laws, we have obtained the very simple result that $L_{m}=V_{m} \Delta t_{m}=0.546 L$, independent of $c_{0}$. This result is remarkable because it does not require any extrapolation with the concentration. This means that the present results are readily valid for the high experimental concentrations. Obviously, the scaling equations for $V_{m}$ and $\Delta t_{m}$ taken separately do involve the copper concentration $c_{0}$. Moreover, their validity range is limited as $L$ increases. However, based on the present results one expects the upper $L$ limit to be rather high for the experimental concentrations. Finally, a good agreement of the scaling equations with the corresponding experimental results has been found and possible physical causes of discrepancy have been identified.

In conclusion, this work suggests that the growth of competing equiaxed dendrites appears as a rather complicated process because it is entirely transient. For this reason, predicting the time evolution of equiaxed dendrite may seem out of reach. However, since our phase-field simulations give scaling laws in the very simplified situation considered here, we can hope to find more general evolution laws corresponding to situations that are closer to 
the experiments. Such scaling evolution laws obviously could be used for higher-scale simulations like mesoscopic envelope models [6].

\section{Acknowledgments}

We would like to thank Laurent Raymond, Claude Arnold and Christophe Kneulé for their help to port our codes to GPU-based computers. We thank the referees and the editors for valuable suggestions that helped us improve the original manuscript. We thank the French Centre for Spatial Research (CNES) for constant financial support.

\section{Appendix A. Supplementary data}

Supplementary data related to this article can be found at http://

\section{References}

[1] Davis JR. Alloying : understanding the basics. Materials Park, $\mathrm{OH}$ : ASM International, 2001.

[2] Kaufman JG and Rooy EL. Aluminum alloy castings : properties, processes, and applications. Schaumburg, IL : American Foundry Soc., 2007.

[3] Dantzig JA and Rappaz M. Solidification. EPFL Press, Switzerland, (2009).

[4] Iqbal N, Van Dijk NH, Offerman SE, Geerlofs N, Moret MP, Katgerman L, and Kearley GJ. In situ investigation of the crystallization kinetics and the mechanism of grain refinement in aluminum alloys. Materials Science and Engineering A, 416:18-32, 2006.

[5] Bogno A, Nguyen-Thi H, Reinhart G, Bilia B, and Baruchel J. Growth and interaction of dendritic equiaxed grains: In situ characterization by synchrotron X-ray radiography. Acta. Mater, 61:1303-1315, 2013.

[6] Souhar Y, De Felice V F, Beckermann C, Combeau H, and Založnik M. Three-dimensional mesoscopic modeling of equiaxed dendritic solidification of a binary alloy. Computational Materials Science, 112:304, 2016. 
[7] Viardin A, Založnik M, Souhar T, and Apel M. Mesoscopic modeling of spacing and grain selection in columnar dendritic solidification: Envelope versus phase-field model. Acta Marerialia, 122:386, 2017.

[8] Chen Y, Li D Z, Billia B, Nguyen Thi H, Qi X B, and Xiao N M. Quantitative phase-field simulation of dendritic equiaxed growth and comparison with in situ observation on $\mathrm{Al}-4 \mathrm{wt} . \% \mathrm{Cu}$ alloy by means of synchrotron X-ray radiography. ISIJ Int., 54:445, 2014.

[9] Gurevich S, Karma A, Plapp M, and Trivedi R. Phase-field study of three-dimensional steady-state growth shapes in directional solidification. Phys. Rev. E, 81 :011603, 2010.

[10] Ghmadh J, Debierre JM, Deschamps J, Georgelin M, Guérin R, and Pocheau A. Directional solidification of inclined structures in thin samples. Acta. Mater., 74:255, 2014.

[11] Bergeon N, Tourret D, Chen L, Debierre JM, Guérin R, Ramirez R, Billia B, Karma A, and Trivedi R. Spatiotemporal dynamics of oscillatory cellular patterns in three-dimensional directional solidification. Phys. Rev. Lett., 11:226102, 2013.

[12] Tourret D, Debierre JM, Song Y, Mota FL, Bergeon N, Guérin R, Trivedi R, Billia B, and Karma A. Oscillatory cellular patterns in threedimensional directional solidification. Phys. Rev. E, 92:042401, 2015.

[13] Pereda J, Mota FL, Chen L, Billia B, Tourret D, Song Y, Debierre JM, Guérin R, Karma A, Trivedi R, and Bergeon N. Experimental observation of oscillatory cellular patterns in three-dimensional directional solidification. Phys. Rev. E, 95:000001, 2017.

[14] Ghmadh J, Debierre J M, Georgelin M, Guérin R, and Pocheau A. Origin and nature of the oscillatory $2 \lambda$-O mode in directional solidification. unpublished, 2018.

[15] Tourret D and Karma A. Three-dimensional dendritic needle network model for alloy solidification. Acta. Mater., 120:240, 2016.

[16] Tourret D and Karma A. Multiscale dendritic needle network model of alloy solidification. Acta. Mater., 61:6474, 2013. 
[17] Chen Y, Bogno AA, Billia B, Kang XH, Nguyen-Thi H, Li DZ, Luo $\mathrm{XH}$, and Debierre JM. Phase-field modeling of the initial transient in directional solidification of Al-4wt.\%Cu alloy. ISIJ Int., 50:1895, 2010.

[18] Steinbach I, Diepers H-J, and Beckermann C. Transient growth and interaction of equiaxed dendrites. J. Cryst. Growth, 275:624, 2005.

[19] Buffet A, Reinhart G, Schenk T, Nguyen Thi H, Gastaldi J, MangelinckNoel N, Jung H, Härtwig J, Baruchel J, and Billia B. Real-time and in situ solidification of Al-based alloys investigated by synchrotron radiation: a unique experimental set-up combining radiography and topography techniques. Phys. Status. Solidi, 204:2721, 2007.

[20] Lu Y, Beckermann C, and Ramirez J C. Three-dimensional phase-field simulations of the effect of convection on free dendritic growth. J. Cryst. Growth, 280:320, 2005.

[21] Karma A. Phase-field formulation for quantitative modeling of alloy solidification. Phy. Rev. Lett., 87:115701, 2001.

[22] Echebarria B, Folch R, Karma A, and Plapp M. Quantitative phase-field model of alloy solidification. Phys. Rev. E, 70:061604, 2004.

[23] Debierre JM, Guérin R, and Kassner K. Crystal growth in a channel: Pulsating fingers, merry-go-round patterns, and seesaw dynamics. Phys. Rev. E, 88 :042407, 2013.

[24] Debierre JM, Guérin R, and Kassner K. Phase-field study of crystal growth in three-dimensional capillaries: Effects of crystalline anisotropy. Phys. Rev. E, 94 :013001, 2016.

[25] Glasner K. Nonlinear preconditioning for diffuse interfaces. J. Comput. Phys., 174 :695, 2001.

[26] Yamanaka A, Aoki T, Ogawa S, and Takak T. GPU-accelerated phasefield simulation of dendritic solidification in a binary alloy. J. Cryst. Growth, 318 :40, 2011.

[27] Karma A and Rappel WJ. Phase-field method for computationally efficient modeling of solidification with arbitrary interface kinetics. Phys. Rev. E, 53:R3017, 1996. 
[28] Karma A and Rappel WJ. Quantitative phase-field modeling of dendritic growth in two and three dimensions. Phys. Rev. E, 57 :4323, 1998.

[29] Hoyt JJ, Asta M, and Karma A. Method for computing the anisotropy of the solid-liquid interfacial free energy. Phys. Rev. Lett., 86 :5530, 2001.

[30] Hultgren R, Desai P R, Hawkins D T, Gleiser M, and Kelley K K. Selected Values of the Thermodynamic Properties of Binary Alloys. (pp 151-153). American Society for Metals, Metals Park, Ohio, 1973.

[31] Smith V G, Tiller W A, and Rutter J W. A mathematical analysis of solute redistribution during solidification. Canad. J. Phys., 33, 1955.

[32] LaCombe JC, Koss MB, and Glicksman ME. Nonconstant tip velocity in microgravity dendritic growth. Phys. Rev. Lett., 83:2997, 1999. 


\section{Figures}

(a)
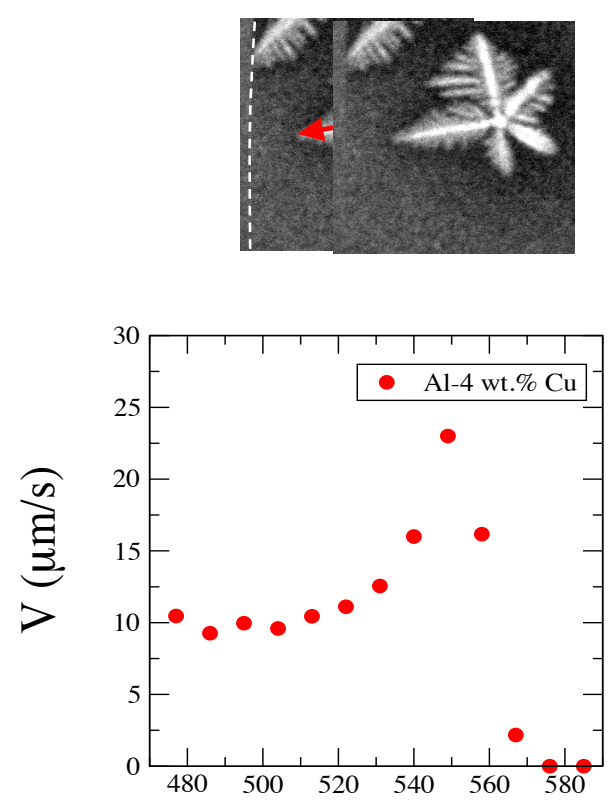

$\mathrm{t}$ (s) (b)
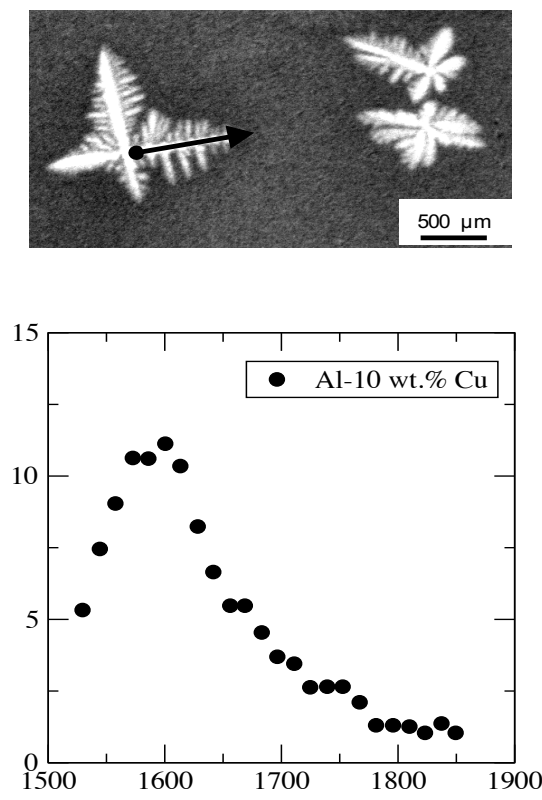

$\mathrm{t}(\mathrm{s})$

Figure 1: Experimental results for the velocity $\mathrm{V}$ of two dendrite arms as a function of time during solidification by isothermal cooling at $R=0.5 \mathrm{~K} / \mathrm{min}$. (a) $\mathrm{Al}-4 \mathrm{wt} \% \mathrm{Cu}$ with a dendrite arm facing a wall. The dashed line on the left indicates the side of the sample. (b) Al-10 wt\% Cu with a dendrite arm facing another dendrite arm. 


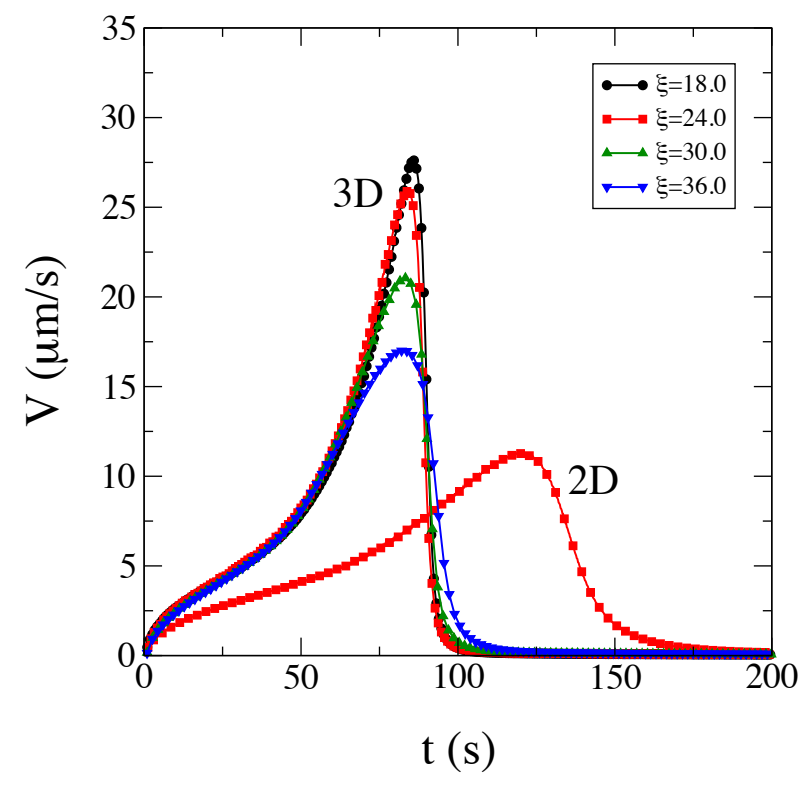

Figure 2: Time evolution of the dendrite tip velocity $\left(L=1000 \mu \mathrm{m}, c_{0}=1.0 \mathrm{wt} \% \mathrm{Cu}\right)$. In $3 \mathrm{D}$, the convergence of the curve with the interface thickness $W_{0}=\xi d_{0}$ is shown for four values of the convergence parameter $\xi$. The difference between the two highest maxima is about 7 percent and almost no evolution is found for $\xi \leq 18.0$. The $2 \mathrm{D}$ results are obtained for $\xi=24.0$ : note the large quantitative differences with the corresponding curve in 3D. 

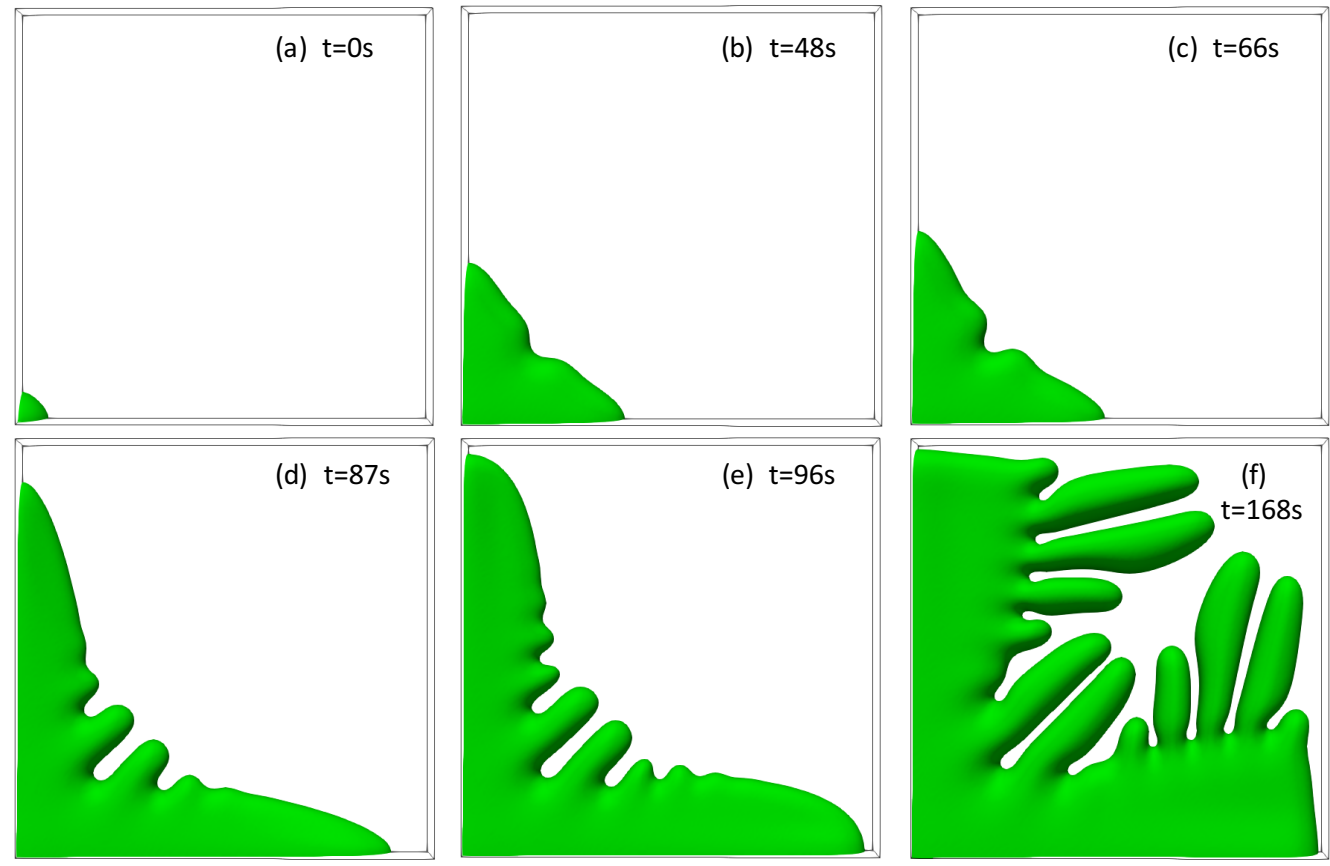

Figure 3: Six snapshots showing the growth of a dendrite simulated in a thin domain of size $1000 \times 100 \times 1000 \mu \mathrm{m}^{3}$ for a copper concentration $c_{0}=1.0 \mathrm{wt} \% \mathrm{Cu}(\psi=0$ isosurfaces are represented). Perspective views are shown to give a better 3D impression: (a) initial condition, (b) slow growth, (c) beginning of the fast growth, (d) end of the fast growth $\left(V=V_{m}\right)$, (e) early ripening, (f) late ripening.
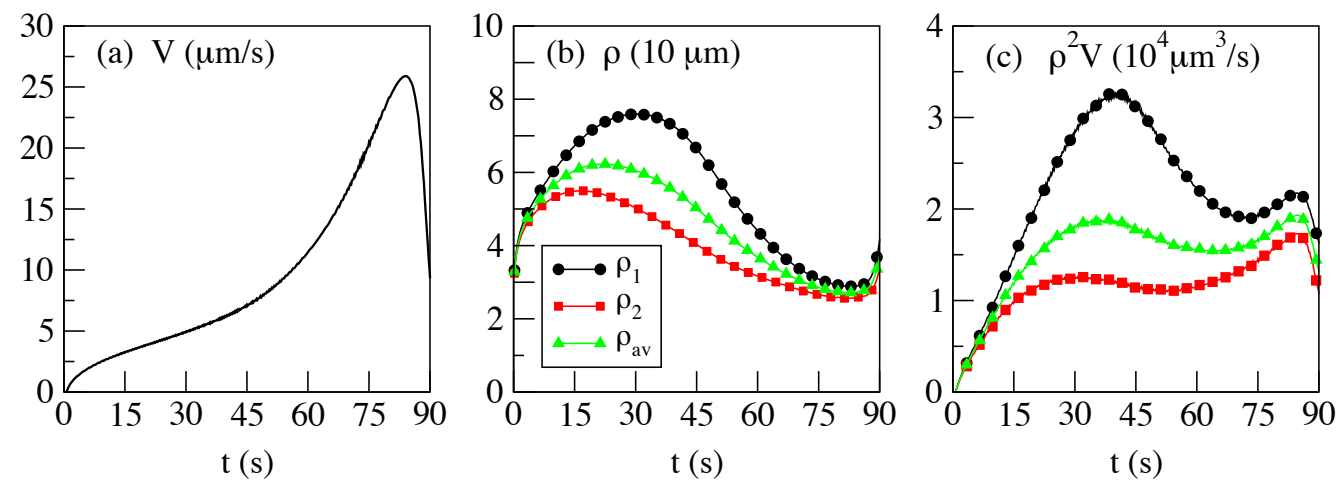

Figure 4: Time evolution of the horizontal tip for the dendrite shown in figure 3: $\rho_{1}\left(\rho_{2}\right)$ is the radius of curvature calculated in the $x z(x y)$ plane and $\rho_{a v}=2\left(\rho_{1} \rho_{2}\right) /\left(\rho_{1}+\rho_{2}\right)$. Plot of (a) tip velocity $V$, (b) tip radius of curvature $\rho$, (c) selection parameter $\rho^{2} V$. $L=1000 \mu \mathrm{m}, c_{0}=1.0 \mathrm{wt} \% \mathrm{Cu}$. 

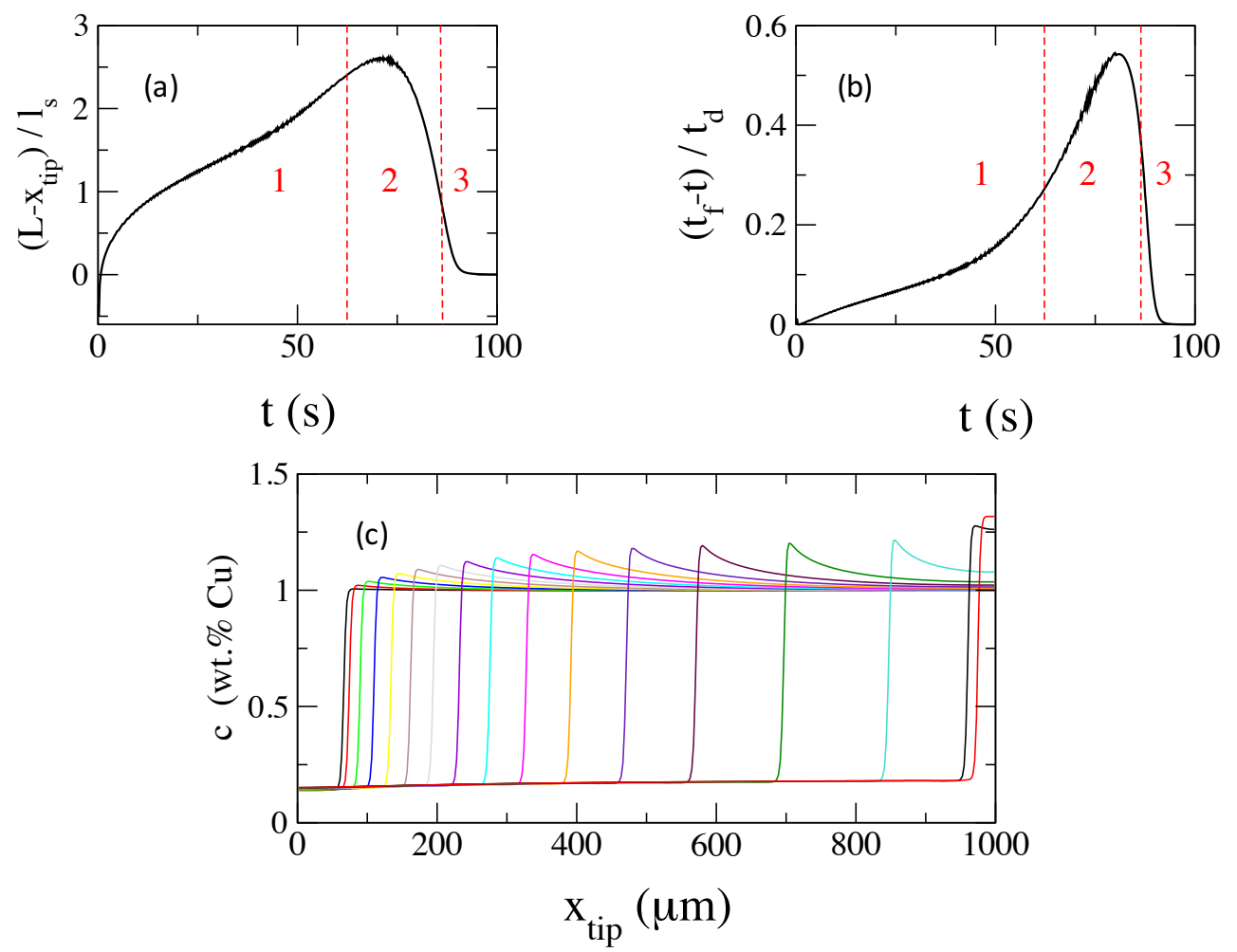

Figure 5: Time evolution of solutal interactions between two colliding dendrite arms. Vertical lines delimit the three growth phases 1, 2 and 3 discussed in the text. (a) ( $L-$ $\left.x_{t i p}\right) / \ell_{s}$, the remaining distance ahead of a dendrite tip divided by the instantaneous diffusion length, as a function of time. (b) $\left(t_{f}-t\right) / t_{d}$, the remaining time divided by the diffusion instantaneous time, as a function of time. (c) Copper concentration profiles along the growth direction $x$ obtained at different times starting from $t=0.6 \mathrm{~s}$ and increasing by steps of $6.0 \mathrm{~s}$ (from the left to the right). $L=1000 \mu \mathrm{m}, c_{0}=1.0 \mathrm{wt} \% \mathrm{Cu}$. 


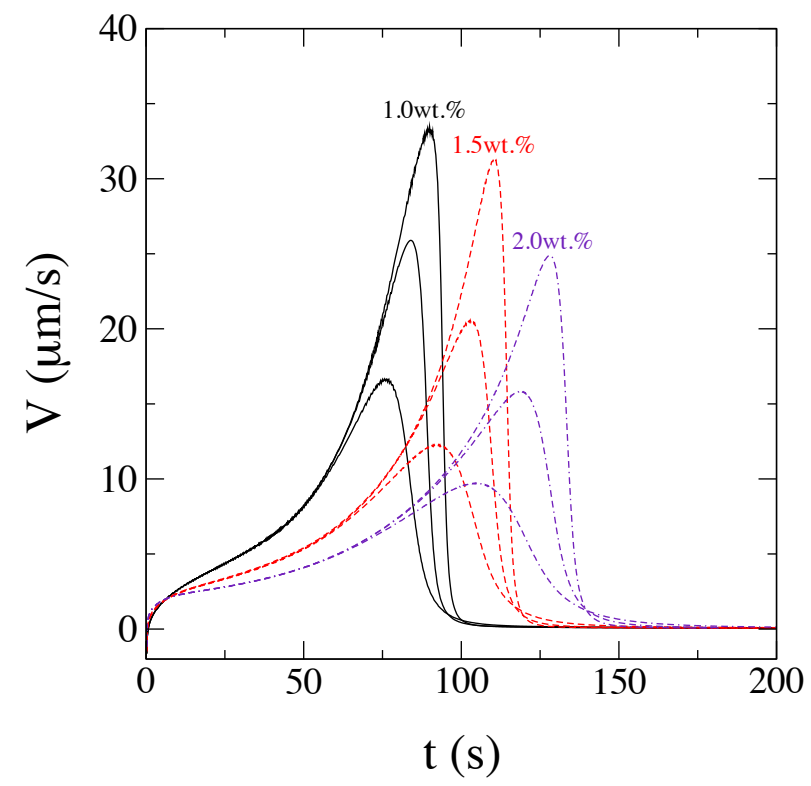

Figure 6: Numerical results for the velocity $V$ of the dendrite tip as a function of time. Three copper concentrations are considered and for each concentration, the simulations are performed for three values of the available distance, $L=1200,1000,800 \mu \mathrm{m}$ from top to bottom. 


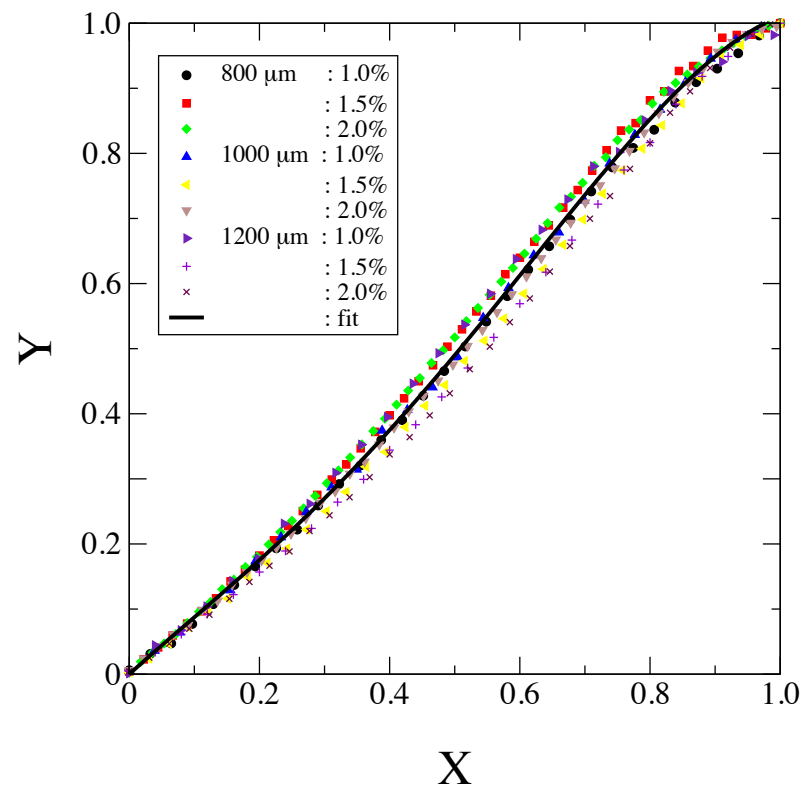

Figure 7: Numerical results for the scaled tip velocity as a function of the scaled time. The data are extracted from the curves of figure 6 . The continuous line is obtained by fitting the data points to a fourth-order polynomial: the result of the fit is $Y=$ $0.90 X-0.43 X^{2}+1.82 X^{3}-1.28 X^{4}$. 


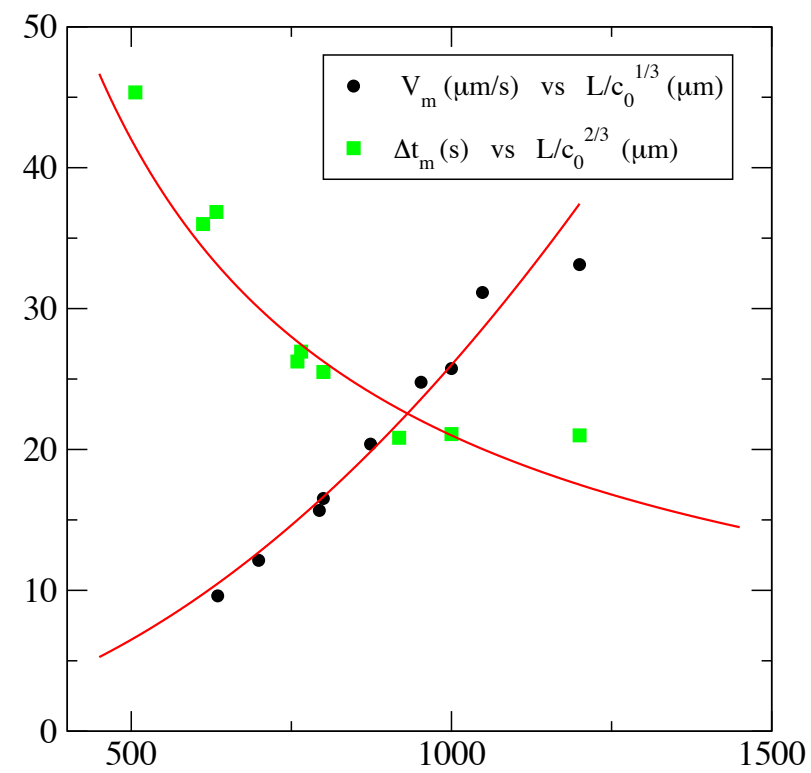

Figure 8: Two scaling equations given in (12) and (14) (lines) that are obtained by fitting the numerical data for the maximum velocity $V_{m}$ (circles) and the time interval $\Delta t_{m}$ (squares). 


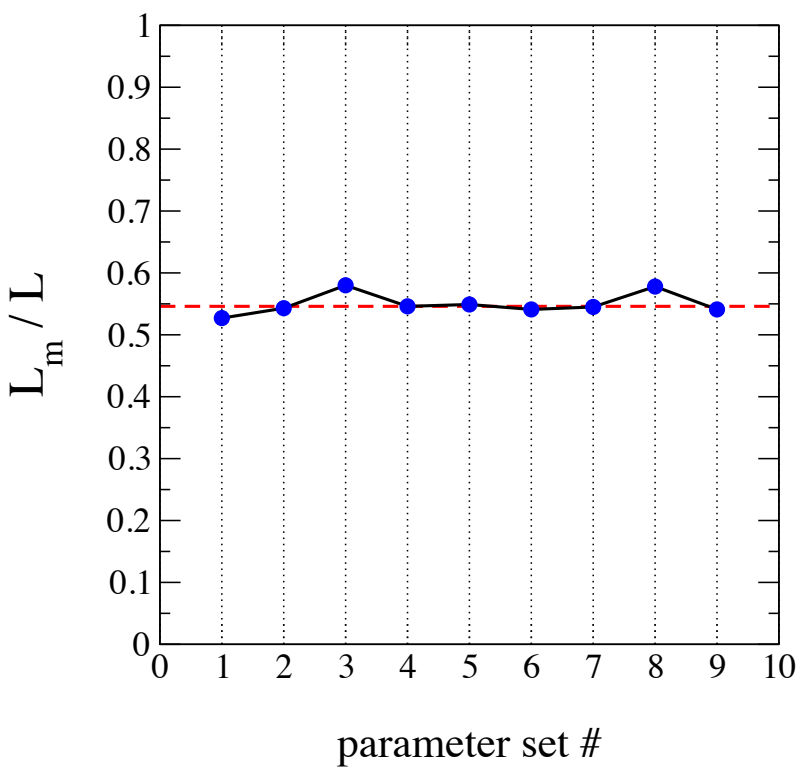

Figure 9: Scaling of the product $L_{m}=V_{m} \Delta t_{m}$ with the available distance $L$. The numerical data (circles) suggest that $L_{m} \simeq 0.546 L$. The parameter sets $\left(L, c_{0}\right)$ imposed in the simulations are numbered from 1 to 9 . They respectively correspond to $\left(L, c_{0}\right)=$ $(800,1),(800,1.5),(800,2),(1000,1),(1000,1.5),(1000,2),(1200,1),(1200,1.5),(1200,2)$, with $L$ in micrometers and $c_{0}$ in wt\% $\mathrm{Cu}$. 